\title{
Incapacidad laboral por diabetes mellitus: características epidemiológicas y complicaciones macro y microangiopáticas más frecuentes
}

\section{Occupational disability due to diabetes mellitus: epidemiological characteristics and macro- and microangiopathic complications more frequent}

\section{Raúl Jesús Regal Ramos}

Recibido: 09-01-14

Aceptado: 11-03-14

\section{Correspondencia}

Raúl Jesús Regal Ramos

Inspector Médico del Instituto Nacional de la Seguridad Social. Dirección Provincial de Madrid

Calle López de Hoyos 169.

28002 Madrid. España.

Teléfono: 915907432

E-mail: raul-jesus.regal@inss.seg-social.es

\section{ResUmen}

La diabetes mellitus (DM) es la enfermedad metabólica más frecuente y conlleva la disminución en la calidad de vida de los sujetos que la padecen, así como la pérdida de años de vida productiva a consecuencia de las complicaciones crónicas o de la mortalidad.

Objetivo: Conocer las características epidemiológicas de los pacientes con invalidez permanente (IP) debida a DM y conocer cuáles son las complicaciones más frecuentes que motivaron la IP y su relación con las distintas ocupaciones.

Método: Se realizó un estudio descriptivo en el que se recogieron pacientes valorados en la Unidad Médica de la Dirección Provincial del INSS de Madrid entre los años 2005 a 2011 con el diagnóstico de diabetes. Se excluyeron aquellos expedientes que no fueron calificados como IP, aquellos que presentaban otras patologías no relacionadas con la diabetes subsidiarias de probable incapacidad (como procesos oncológicos, demencia, esclerosis múltiple,..) y los casos de diabetes secundarias. Se estudian las variables edad, sexo, estado civil, profesión, régimen de afiliación a la Seguridad Social, y las recogidas en el apartado diagnóstico del informe médico de síntesis (tipo de diabetes, complicaciones derivadas de la DM y factores de riesgo cardiovascular).

Resultado: El total de pacientes estudiados fue de 896. Respecto a la población general ocupada de nuestro entorno observamos un 31\% más de varones ( $p<0,0005$; Razón de prevalencia $=1,56)$ y un 28\% más de ocupaciones del grupo VII de la CNO-11 (industria y construcción) $(\mathrm{p}<0,005$; Razón de prevalencia =2,3). El 48\% de las profesiones científicas y el $36 \%$ de las administrativas estaban diagnosticados de oftalmopatía. El 39\% de trabajadores de industria y construcción estaban diagnosticados de algún grado de coronariopatía.

Conclusión: Nuestros resultados permiten objetivar que la oftalmopatía es la complicación que más se asocia a invalidez permanente en profesiones de alta exigencia visual y la coronariopatía en las profesiones de esfuerzo físico. Además permiten lanzar la hipótesis de que ser varón y tener una profesión relacionada con la industria o la construcción aumentan el riesgo de obtener una IP por complicaciones de la diabetes.

Palabras clave: Incapacidad. Diabetes Mellitus, Medicina de trabajo. 
Abstract

Diabetes mellitus is the most common metabolic disease and leads to decreased quality of life of individuals with the disease, and the loss of years of productive life as a result of chronic complications or mortality.

Objective: To determine the epidemiological characteristics of patients with permanent disability due to diabetes mellitus and to know which are the most common complications that led to the permanent disability and its relationship with different occupations.

Method: This was a descriptive study that evaluated patients who were collected in the Medical Unit of the Provincial Directorate of INSS of Madrid between 2005-2011 with a diagnosis of diabetes. It excluded those records that were not classified as IP, those who had other diseases unrelated to diabetes likely subsidiaries disability (as oncological processes, dementia, multiple sclerosis, ..) and secondary diabetes cases. We study the variables age, sex, marital status, occupation, type of affiliation to the Social Security and those listed in paragraph diagnostic of specific medical report (type of diabetes, complications of DM and cardiovascular risk factors).

Result: The number of patients studied was 896 . Regarding the general population occupied in our environment we see a $31 \%$ increase in males $(\mathrm{p}<0.0005$; prevalence ratio $=1.56)$ and $28 \%$ more than the group VII of the CNO -11 (industry and construction) $(\mathrm{p}<0.005$; prevalence ratio $=2.3$ ). $48 \%$ of the scientific professions and $36 \%$ of administrative ophthalmopathy were diagnosed. $39 \%$ of industry and construction workers were diagnosed with some degree of coronary artery disease.

Conclusion: Our results objectively in our ophthalmopathy is the most frequent complication associated with permanent disability in high-demand professions visual and coronary artery disease in the professions of physical effort. They also allow to hypothesize that being male and having a profession related to the construction industry or increase the risk of getting a permanent disability for diabetes complications.

Keywords: Disability. Diabetes Mellitus, occupational medicine.

Med Segur Trab (Internet) 2014; 60 (234) 99-107 


\section{INTRODUCCIÓN}

La diabetes mellitus (DM) es la enfermedad metabólica más frecuente. El estudio di@bet.es, de publicación reciente, estima que la prevalencia de DM tipo 2 en la población española es de un 13,79\% (intervalo de confianza [IC] del 95\% 12,84-14,74) ${ }^{1}$. Dicho estudio establece además una prevalencia del 14,8\% para los estados prediabéticos (glucemia basal alterada e intolerancia a la glucosa). Por tanto, se contempla como un problema de salud pública, que establece un alto impacto económico y social, que conlleva la disminución en la calidad de vida de los sujetos que la padecen, así como la pérdida de años de vida productiva a consecuencia de las complicaciones crónicas o de la mortalidad.

Las complicaciones crónicas de la diabetes son responsables de la mayoría de hospitalizaciones de los pacientes diabéticos, que representan el 55\% del gasto total según el estudio CODE- $2^{2}$. Dicho estudio recoge también que el coste anual medio de un paciente sin complicaciones es de 883 euros frente a 1.403 de un paciente con complicaciones microvasculares, 2.022 cuando existían complicaciones macrovasculares y 2.133 cuando coexistían ambos tipos de complicaciones ${ }^{3}$.

Otro estudio realizado por la London School of Economics ${ }^{4}$ indica que España es el segundo país, de los cinco más poblados de la Unión Europea, con más costes indirectos (los relacionados con la disminución o pérdida de productividad debida a la mortalidad prematura y a la incapacidad laboral) asociados a la diabetes, 17.630 millones de euros al año, por debajo solo de Alemania. De estos 17.630 millones, más de 9.000 se deben a sus jubilaciones anticipadas.

Se define como invalidez permanente (IP) a la situación del trabajador que tras estar sometido al tratamiento prescrito presenta reducciones anatómicas graves, susceptibles de determinación objetiva y previsiblemente definitivas, que disminuyen o anulan su capacidad laboral $^{5}$. Los equipos de valoración de incapacidades (EVI), encuadrados en cada Dirección Provincial del Instituto Nacional de la Seguridad Social (INSS), son los que examinan la posible situación de incapacidad del trabajador y formulan los dictámenes con sus conclusiones ${ }^{6}$. El dictamen de estos EVIs se apoya en un informe médico realizado por el médico inspector del INSS, en los informes realizados por los médicos del Servicio Público de Salud, en los antecedentes profesionales y en los informes de cotización que condicionan el acceso al derecho.

La DM, por sí misma, no debe considerarse como una enfermedad intrínsecamente incapacitante. En ausencia de complicaciones y con un control metabólico bueno los problemas laborales son mínimos ${ }^{7}$, salvo en profesiones específicamente reguladas.

\section{JUSTIFICACIÓN DEL ESTUDIO}

La diabetes tiene una elevada incidencia y unos costes personales y económicos muy altos, sin embargo, no existen estudios sobre los aspectos de la diabetes más invalidantes desde el punto de vista laboral en pacientes de nuestro ámbito.

\section{OBJETIVO} Mellitus.

Conocer las características epidemiológicas de los pacientes con IP debida a Diabetes

Conocer cuáles son las complicaciones más frecuentes que motivaron la IP y su relación con las distintas ocupaciones. 


\section{MÉTODO}

Los expedientes de IP constan entre su documentación de un "informe médico de síntesis" (IMS) realizado por los médicos inspectores del INSS que recoge y resume la clínica, exploraciones, pruebas complementarias, patologías, tratamientos y la valoración funcional y laboral de estos pacientes. Dicho informe consta de varios apartados, siendo uno de los principales el reservado para el diagnóstico. De este apartado diagnóstico recabaremos la información médica de este estudio.

Se incluyeron todos los pacientes valorados en la Unidad Médica de la Dirección Provincial del INSS de Madrid entre los años 2005 a 2011 en cuyo IMS figurase el diagnóstico de diabetes y términos relacionados (diabético, diabética, DM, insulinodependiente, glucemia, hipoglucemia, hiperglucemia y glucosa). Por limitaciones informáticas solo fueron seleccionados los expedientes valorados antes de agotar el periodo máximo de incapacidad temporal (expedientes iniciados a petición del paciente, del servicio público de salud, de la mutua o del propio INSS). No se valora un volumen superior compuesto por expedientes llegados al plazo máximo de Incapacidad Temporal (IT), expedientes internacionales, expedientes de MUFACE, reclamaciones, revisiones, etc.

Se excluyen aquellos expedientes que no fueron calificados como IP, aquellos que presentaban otras patologías no relacionadas con la diabetes subsidiarias de probable incapacidad (como procesos oncológicos, demencia, esclerosis múltiple,..) y los casos de diabetes secundarias (ver figura 1).

Figura 1

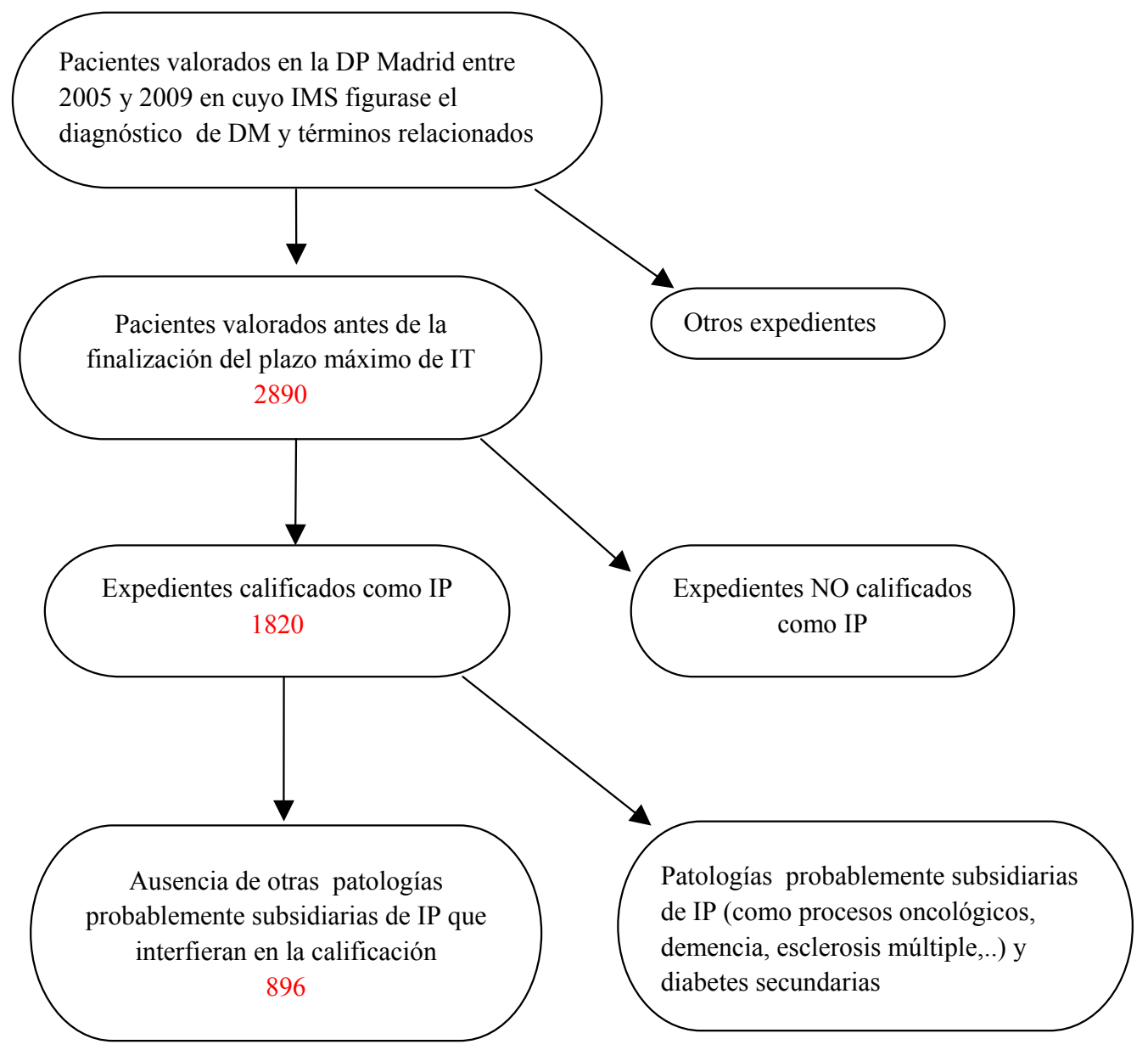


En todos los casos se analizaron las siguientes variables edad, sexo, estado civil, profesión, régimen de afiliación a la Seguridad Social, y las recogidas en el apartado diagnóstico del IMS (tipo de diabetes, complicaciones derivadas de la DM y factores de riesgo cardiovascular, FRCV).

El estadio de las complicaciones y el tiempo devolución de la diabetes no fueron recogidos en el estudio dado que no existe uniformidad en la forma de expresarlos en los IMS.

Para establecer la variable ocupación profesional se utilizó la aplicación AYUDACOD del Instituto Nacional de Estadística (INE), de modo que al introducir la profesión de cada paciente este programa nos dio un código que se encuadró en uno de los 10 grandes grupos de la Clasificación Nacional de Ocupaciones del año 2011 (CNO-11).

Posteriormente se comparó la prevalencia de las variables sexo y profesión en nuestra muestra y en la población general. Los datos de la población general se obtuvieron del Instituto Nacional de Estadística (INE), Encuesta de Población Activa-Ocupados.

Fuente primaria de información: Unidad de informática de la Dirección Provincial del Instituto Nacional de la Seguridad Social de Madrid.

Para el procesamiento de los datos y los cálculos estadísticos se creó una base de datos con Microsoft Excel.

\section{CONSIDERACIONES ÉTICAS}

El presente estudio se ha realizado con la autorización de la Dirección General del Instituto Nacional de la Seguridad Social.

La información perteneciente a los pacientes integrantes del estudio se ha tratado de acuerdo a lo establecido en la Ley Orgánica 15/1999 de protección de datos personales.

\section{RESULTADOS}

El total de pacientes estudiados fue de 896 (ver figura 1).

La tabla I recoge las características epidemiológicas de la muestra

Tabla I

\begin{tabular}{|c|c|c|}
\hline \\
\hline \multicolumn{2}{|c|}{ Variable } & \multirow{2}{*}{$\begin{array}{c}\text { Total } \\
896\end{array}$} \\
\hline Pacientes calificado con IP* & & \\
\hline \multirow[t]{2}{*}{ Sexo } & Mujeres & $124(14 \%)$ \\
\hline & Varones & $772(86 \%)$ \\
\hline \multirow[t]{7}{*}{ AÑO } & 2005 & $127(14 \%)$ \\
\hline & 2006 & $181(20 \%)$ \\
\hline & 2007 & $139(15 \%)$ \\
\hline & 2008 & $137(15 \%)$ \\
\hline & 2009 & $112(12 \%)$ \\
\hline & 2010 & $103(11 \%)$ \\
\hline & 2011 & $97(10 \%)$ \\
\hline \multirow[t]{5}{*}{ EDAD } & media & \\
\hline & $21-25$ & 1 \\
\hline & $26-30$ & 2 \\
\hline & $31-35$ & 9 \\
\hline & $36-40$ & 19 \\
\hline
\end{tabular}




\begin{tabular}{|c|c|c|}
\hline \multicolumn{2}{|c|}{ Variable } & \multirow[t]{2}{*}{ Total } \\
\hline EDAD & media & \\
\hline & $41-45$ & 41 \\
\hline & $46-50$ & 77 \\
\hline & $51-55$ & 129 \\
\hline & $56-60$ & 276 \\
\hline & $61-65$ & 258 \\
\hline & $66-70$ & 10 \\
\hline \multirow[t]{4}{*}{ Estado civil } & Casado & $569(63 \%)$ \\
\hline & & Mujeres 58 \\
\hline & & Varones $511(86 \%)$ \\
\hline & No casado & \\
\hline \multirow[t]{3}{*}{ Régimen SS S** $^{* *}$} & Régimen general & 686 \\
\hline & Autónomos & 196 \\
\hline & Otros & 14 \\
\hline
\end{tabular}

El 86\% de la muestra eran varones, un 31\% más que los datos del INE acerca de la población activa general $(\mathrm{p}<0,0005$; Razón de prevalencia $=1,56)$.

En los últimos 6 años se observa un descenso de los casos. Aunque se trata de un periodo de observación corto, esta tendencia podría estar en relación con la mejora de los tratamientos que retrasan la aparición de complicaciones graves a una edad posterior a la vida laboral.

Casi 2/3 de las valoraciones se realiza entre los 55 y 65 años, como ocurre en otras patologías crónicas. La mediana de edad de la muestra de 57 años.

El 63\% de la muestra estaba casada.

El 76\% pertenecían al Régimen General y el 22\% al de Autónomos.

Las ocupaciones del grupo VII (Ver tabla II) de la CNO-11 (industria y construcción) son los que más prevalecen, 28\%, más del doble de los datos que nos da la Encuesta de Población Activa - Ocupados del INE ( $p<0,005$; Razón de prevalencia =2,3). Les siguen las ocupaciones del grupo V y del grupo IX. La Comunidad de Madrid tiene una proporción menor de agricultores y ganaderos que el resto de estado español, lo que explica el escaso porcentaje de la muestra. EL $0 \%$ de pacientes de las fuerzas armadas se explica porque la invalidez de estos pacientes se tramita en otras instituciones distintas al INSS.

Tabla II. Clasificación Nacional de Ocupaciones 2011

\begin{tabular}{rrrr}
\hline & & Muestra & $\begin{array}{c}\text { Población } \\
\text { general }\end{array}$ \\
\hline Ocupación & I. Dirección de las empresas y de las administraciones & $1 \%$ & $5 \%$ \\
& públicas & & \\
II. Técnicos y profesionales científicos e intelectuales & $4 \%$ & $18 \%$ \\
III. Técnicos y profesionales de apoyo & $5 \%$ & $12 \%$ \\
IV. Empleados de tipo administrativo & $10 \%$ & $11 \%$ \\
V. Trabajadores de los servicios de restauración, & $24 \%$ & $21 \%$ \\
$\quad$ personales, protección y vendedores de los comercios & & \\
VI. Trabajadores cualificados en la agricultura y en la pesca & $<1 \%$ & $2 \%$ \\
VII. Artesanos y trabajadores cualificados de las industrias & $28 \%$ & $12 \%$ \\
$\quad$ manufactureras, la construcción, y la minería, excepto & & \\
$\quad$ los operadores de instalaciones y maquinaria & & $8 \%$ \\
VIII. Operadores de instalaciones y maquinaria, y montadores & $12 \%$ & $14 \%$ & $10 \%$ \\
IX. Trabajadores no cualificados (="peones") & $0 \%$ & $1 \%$ \\
X. Fuerzas armadas & &
\end{tabular}


El 86\% de la muestra estaban diagnosticados de DM II y el 14\% de tipo I. El FRCV más recogido en el apartado diagnóstico del IMS fue el de HTA (ver tabla III).

Tabla III

\begin{tabular}{llccc}
\hline & \multicolumn{1}{c}{ Variable } & Total & Mujeres & Hombres \\
\hline Tipo de DM & DM I & $125(14 \%)$ & & \\
& DM II & $771(86 \%)$ & & \\
\hline FRCV & Obesidad & $110(12 \%)$ & 21 & 89 \\
& HTA & $297(33 \%)$ & 29 & 268 \\
& Dislipemia & $163(18 \%)$ & 14 & 149 \\
\hline
\end{tabular}

Las complicaciones derivadas de la diabetes más prevalentes son la oftalmopatía y la cardiopatía.

La Figura 2 describe la relación entre las complicaciones y la ocupación (grupos CNO-11). El grupo VI lo componen solo 4 casos por lo que se decide no reflejarlos en el gráfico. El 10,6\% de la muestra presentaba algún tipo de amputación consecuencia de la DM, observándose una frecuencia mayor en los trabajos que requieren menor cualificación.

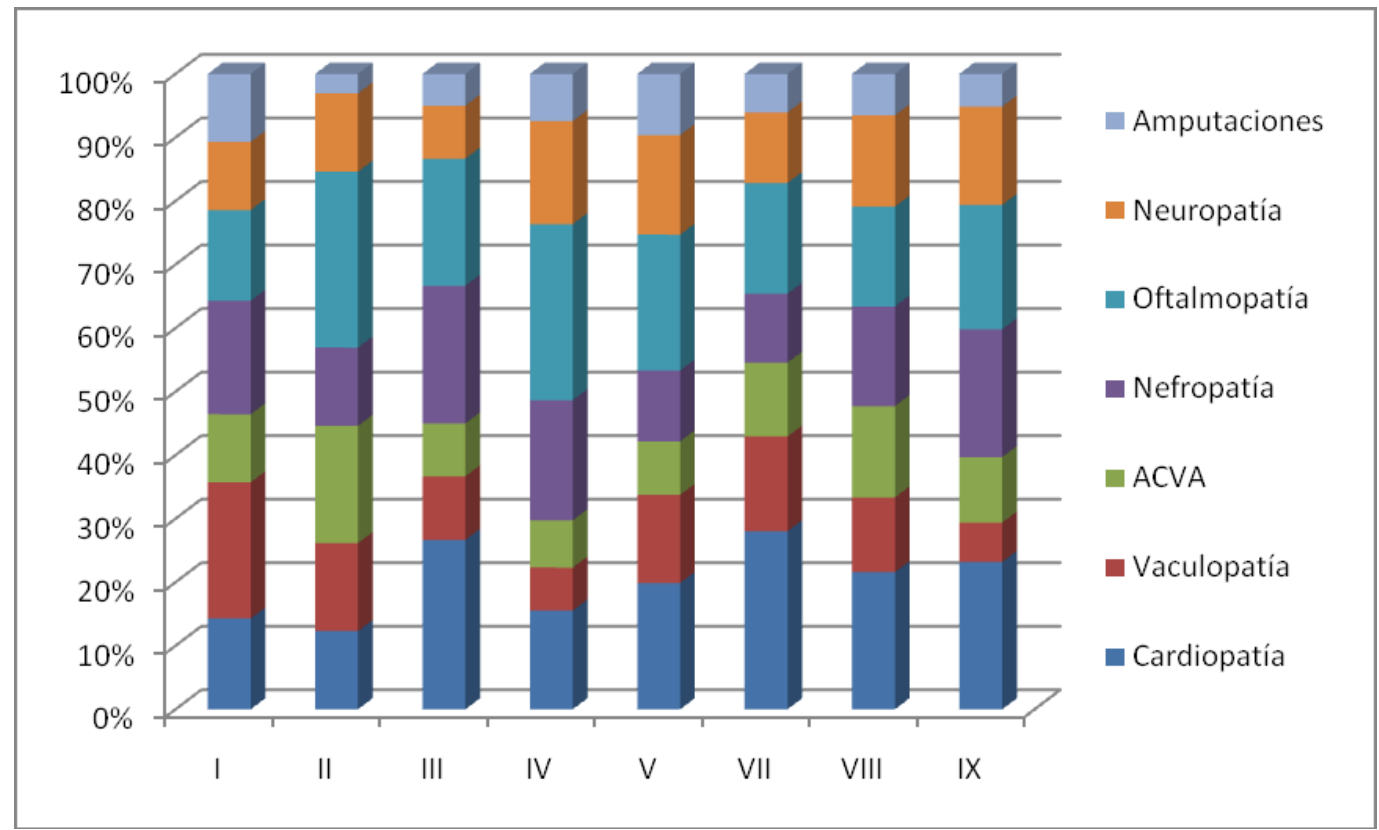

\section{DISCUSIÓN}

Como era esperable, nuestros resultados confirman que la oftalmopatía es la complicación que se asocia con más frecuencia a profesiones científicas y administrativas (CNO-11 II y IV), mientras que la coronariopatía es la complicación que más se asocia a "trabajos de industria y construcción" y a "otros trabajos no cualificados" (CNO-11 VII y IX). La oftalmopatía también ha sido la complicación más frecuente asociada a invalidez por DM en otros estudios ${ }^{8}$.

La oftalmopatía es la complicación que aparece antes y la coronariopatía la que más IP genera, por este motivo es normal que ambas sean las que con más frecuencia se recogen en los IMS. La nefropatía, al igual que ocurre con la vasculopatía y la neuropatía son complicaciones que se convierten en severas de una forma más tardía, y por este motivo, menos frecuentes que la oftalmopatía o la coronariopatía en la edad laboral ${ }^{\text {. }}$. 
El porcentaje de diabéticos tipo I y tipo II es similar al de la población general ${ }^{10}$.

La importancia de la HTA en el desarrollo de complicaciones de la DM está claramente establecida. Ya el UKPDS en el año $2000^{11}$ demostró que el control de la TA es más importante que el control glucémico estricto en la prevención de eventos cardiovasculares y la $\mathrm{ADA}^{12}$ lo destaca como un objetivo terapéutico primordial. Nuestro estudio demuestra que no solo es el FRCV más frecuente en la DM con complicaciones sino que es el más importante para que dichas complicaciones alcancen una gravedad susceptible de IP.

En nuestra muestra de pacientes con IP, observamos una prevalencia de varones (Razón de Prevalencia: 1,56) y de trabajadores de la industria y construcción (Razón de Prevalencia 2,3) significativamente más elevada que la población activa de nuestro entorno. Nuestros resultados permiten lanzar la hipótesis de que el sexo y la ocupación pueden comportarse como factores predictores de obtención de IP en estos pacientes. El hecho de que sean los varones los que realizan la mayoría de trabajos de construcción podría actuar como factor de confusión, pero este aumento del porcentaje de varones se aprecia en todas las ocupaciones.

\section{LIMITACIONES DEL ESTUDIO}

Se trata de un estudio descriptivo y por tanto será necesario realizar otros estudios de cohortes bien diseñados con períodos de seguimiento amplios para confirmar nuestros resultados.

Aunque la muestra solo está compuesta por expedientes iniciados antes del plazo máximo de los 12 meses, sigue siendo representativa de la población que valoramos.

El hecho de que el estudio incluya solo los datos médicos recogidos en el apartado diagnóstico del IMS puede conllevar pérdida de información (complicaciones subclínicas, estadios incipientes, FRCV recogidos en el IMS pero no en el apartado diagnóstico) pero asegura la inclusión de los datos más relevantes.

La influencia de otras enfermedades en la calificación de la IP se minimiza al retirar los casos de patologías no relacionadas con la DM subsidiarias de probable incapacidad (procesos oncológicos, demencia, esclerosis múltiple,...).

La influencia del trabajo en la calificación de la IP no puede evitarse, dado que es una variable intrínsecamente unida a dicha calificación. Solo en los casos en los que la calificación fuese la de incapacidad para todos los trabajos, dicha variable pasaría a un segundo plano, pero el presente estudio no distingue entre IP para la profesión que desempeña el trabajador o IP para todas las profesiones.

\section{CONCLUSIONES}

En las profesiones de alta exigencia visual ("científicas" y "administrativas") con IP la complicación más frecuente es la oftalmopatía, mientras que entre las profesiones de mayor esfuerzo físico ("trabajos de industria y construcción" y "otros trabajos no cualificados”) con IP la complicación más frecuente es la coronariopatía.

Este estudio además permite lanzar la hipótesis de que ser varón y tener una profesión relacionada con la industria o la construcción aumentan la posibilidad de recibir una IP por complicaciones de la diabetes.

Pese a las limitaciones descritas del presente trabajo, la escasa cantidad de estudios al respecto, el hecho de que las variables recogidas sean las más relevantes y el hecho de tener una muestra representativa nos permite obtener unas conclusiones fiables e interesantes para futuros proyectos que analicen las repercusiones socioeconómicas derivadas de la diabetes. 


\section{AGRADECIMIENTOS}

A la Unidad Médica y a la Unidad de Informática de la Dirección Provincial de Madrid y a la Subdirección General de Coordinación de Unidades Médicas del INSS, sin cuya colaboración y apoyo no hubiera sido posible la realización de este trabajo.

\section{REFERENCIAS BIBLIOGRÁFICAS}

1. Soriguer F, Goday A, Bosch-Comas A, Bordiú E, Calle-Pascual A, et al. Prevalence of diabetes mellitus and impaired glucose regulation in Spain: the Di@bet.es Study. Diabetologia. 2012;55:88-93.

2. Massi-Benedetti M. The cost of diabetes Type II in Europe: the CODE-2 Study. Diabetologia. 2002;45:S1S4.

3. Mata M, et al. El coste de la diabetes tipo 2 en España. El estudio CODE-2 Gac Sanit 2002;16(6):511-20.

4. Panos Kanavos, Stacey van den Aardweg and Willemien Schurer. Diabetes expenditure, burden of disease and management in 5 EU countries. LSE Health, London School of Economics. January 2012.

5. Texto Refundido de la Ley General de la Seguridad Social (Real Decreto Legislativo 1/1994, de 20 de junio). BOE n. ${ }^{\circ}$ 154, de 29/6/1994); Art. 136.

6. Real Decreto $1300 / 1995$, de 21 de julio, por el que se desarrolla, en materia de incapacidades laborales del sistema de la Seguridad Social, la Ley 42/1994, de 30 de diciembre, de medidas fiscales, administrativas y de orden social. BOE n. ${ }^{\circ} 198$, de 19 de agosto de 1995; 25.856.

7. Vicente-Herrero M.T., et al. Minusvalía e incapacidad en la diabetes y sus complicaciones. Una revisión desde la legislación española. Av Diabetol. 2010;26:451-6.

8. Reyes Chacón MT et al. Invalidez laboral por diabetes mellitus tipo 2: experiencia en 393 pacientes de Ciudad Juárez, México. Archivos en Medicina Familiar Vol. 12 Núm 4 Octubre-Diciembre 2010.

9. Barrot J., González A. Diabetes mellitus: actualización, novedades y controversias. AMF 2012;8(3):124134.

10. Cabezas J, Casanueva F. Tratamiento de la hiperglucemia en la diabetes mellitus tipo 2: hacia un tratamiento individualizado. Medicine 2011;10(92):6230-9.

11. Stratton IM et al. Association of glycaemia with macrovascular and microvascular complications of type 2 diabetes (UKPDS 35): prospective observational study. BMJ 2000; 321: 405-412.

12. American Diabetes Asssociation. Standards of medical care in diabetes-2011. Diabetes Care. 2011;34Suppl1: S11-61. 